\title{
Structural Characterisation of SARM in Axon Degeneration and Cell Death
}

Shane Michael Horsefield ${ }^{1}$, Thomas Ve ${ }^{2}$, Xiaoxiao Zhang ${ }^{3}$, Lachlan Casey ${ }^{1}$, Bostjan Kobe ${ }^{1}$

${ }^{1}$ School of Chemistry and Molecular Biosciences, The University Of Queensland, Brisbane, Australia, ${ }^{2}$ Institute for Glycomics, Griffith University, Gold Coast, Australia, ${ }^{3}$ Commonwealth Scientific and Industrial Research Organisation, Canberra, Australia E-mail: s.horsefield@uq.edu.au

Degeneration of axons eliminates unwanted or damaged nerves from an organism as part of normal neuronal development and injury, but is also a common feature in neurodegenerative disease and neuropathies. Recently, a Toll-like receptor (TLR) adaptor protein, sterile-alpha and armadillo motif-containing protein (SARM), has shown to promote axon degeneration after injury (Wallerian degeneration) and promote cell death. The protein comprises three domains: two central tandem sterile-alpha motifs (SAM) flanked by an N-terminal armadillo repeat motif (ARM) and a C-terminal Toll/interleukin-1 receptor (TIR) domain. We have solved the crystal structure of the tandem SAM domains of human SARM at $2.8 \AA$ resolution which form an octameric ring. Using small-angle X-ray scattering (SAXS) and multi-angle light scattering (MALS) we can determine this ring structure is conserved across species. We have also solved the crystal structure of the C-terminal TIR domain at $1.8 \AA$ resolution. The TIR domains are responsible for transmitting signal in TLR signalling and requires a clustering event to bring TIR domains in close proximity. In relation to SARM promoting axon degeneration and cell-death, we hypothesise that the tandem SAM domains act as the "clustering mechanism" to bring the TIR domains together, which then recruits downstream proteins to cause axon degeneration and cell death. We are currently testing mutants that knock out the oligomeric ring of the tandem SAM domains and residues in the TIR domain to analyse the role these have in axon degeneration and cell-death, which could provide new targets for therapeutic drugs in neurodegenerative disease and neuropathies. This concept of forming complex assemblies in immunity and inflammation are seen in other immune pathways, such as the inflammasome pathway, effector-triggered immunity in plants and apoptosis.

[1] Gerdts, J. et. al,. (2013) JNeuroSci 33, 13569-13580.

[2] Panneerselvam, P., and Ding, J.L. (2015) Int. Rev. Immunol, 34, 432-444.

[3] Veriepe, J. et. al,. (2015). Nat. Comm, 6, 7319.

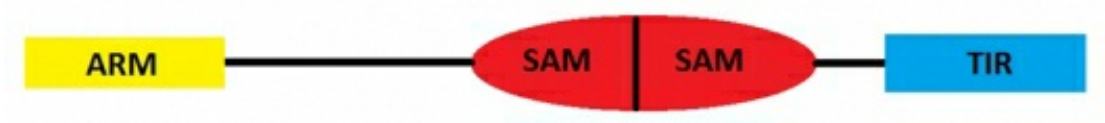

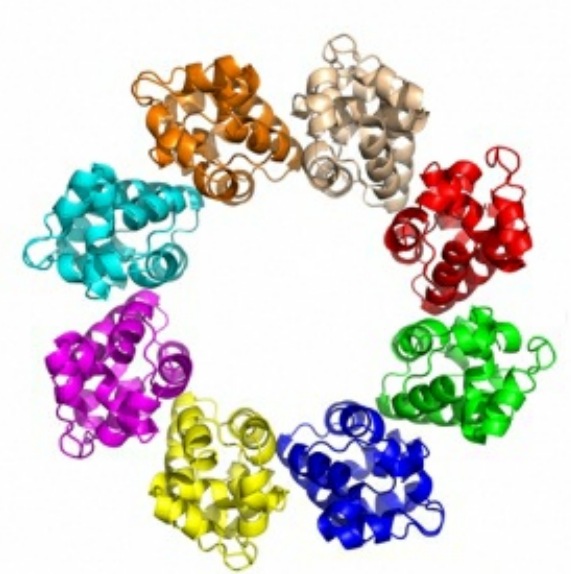

Tandem SAM domains octamer formation

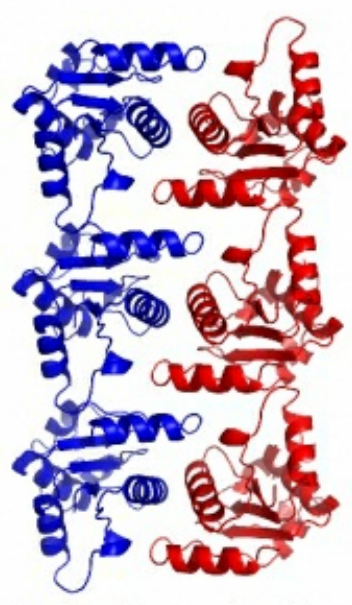

TIR domain crystal packing

Keywords: Immunology, Structural Biology, Cell Death 